\title{
Dislocation Mechanics Pile-Up and Thermal Activation Roles in Metal Plasticity and Fracturing
}

\author{
Ronald W. Armstrong
}

Center for Engineering Concepts Development, Department of Mechanical Engineering, University of Maryland, College Park, MD 20742, USA; rona@umd.edu; Tel.: +1-410-723-4616

Received: 14 January 2019; Accepted: 27 January 2019; Published: 31 January 2019

\begin{abstract}
Dislocation pile-up and thermal activation influences on the deformation and fracturing behaviors of polycrystalline metals are briefly reviewed, as examples of dislocation mechanics applications to understanding mechanical properties. To start, a reciprocal square root of grain size dependence was demonstrated for historical hardness measurements reported for cartridge brass, in line with a similar Hall-Petch grain size characterization of stress-strain measurements made on conventional grain size and nano-polycrystalline copper, nickel, and aluminum materials. Additional influences of loading rate (and temperature) were shown to be included in a dislocation model thermal activation basis, for calculated deformation shapes of impacted solid cylinders of copper and Armco iron materials. Connection was established for such grain size, temperature, and strain rate influences on the brittle fracturing transition exhibited by steel and other related metals. Lastly, for AISI 1040 steel material, a fracture mechanics based failure stress dependence on the inverse square root of crack size was shown to approach the yield stress at a very small crack size, also in line with a Hall-Petch dependence of the stress intensity on polycrystal grain size.
\end{abstract}

Keywords: dislocation mechanics; yield strength; grain size; thermal activation; strain rate; impact tests; brittleness transition; fracturing; crack size; fracture mechanics

\section{Historical Background Leading to Dislocation Mechanics}

A substantial improvement to the strength properties of metals by means of refining their internal crystal or grain size has been known for centuries [1]. An example is shown in Figure 1 of early 20th century measurements on the Brinell Hardness Number (BHN) of alpha brass materials, being shown in later work to follow a reciprocal square root of grain diameter dependence [2].

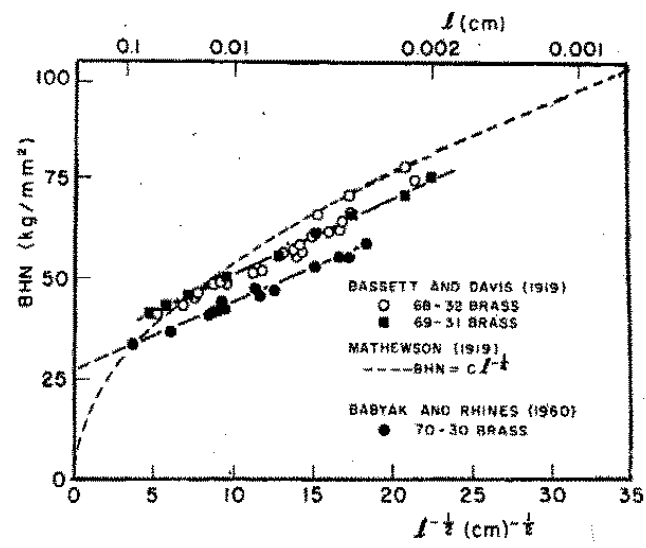

Figure 1. The Brinell Hardness Number $(\mathrm{BHN})$ of alpha brass materials as a function of the reciprocal square root of polycrystalline grain size, $\ell^{-1 / 2}$; see Reference [2] for references, $1.0 \mathrm{~kg} / \mathrm{mm}^{2}=9.81 \mathrm{MPa}$. 
In Figure 1, the BHN is defined by the load applied to a ball indenter divided by the surface area of the (assumed) spherical cap of the residual permanent indentation. The hardness is more often specified as a Meyer hardness $(\mathrm{MH})$, for which the projected surface area of the residual indent is employed. The MH would show essentially the same grain size dependence as has now been established for many metals and alloys.

The hardness relates to the compressive or tensile flow stress at true strain, $\varepsilon$, through the relationship: $\mathrm{MH} \approx 3 \sigma_{\varepsilon}$ and $\varepsilon=\sim 7.5 \%$. Thus, explanation of a grain size dependent hardness follows from association of the hardness with a unidirectional flow stress in which grain size dependence, known as a Hall-Petch relationship, has been explained in terms of dislocation pile-ups in slip bands behaving similarly to shear cracks when blocked at grain boundaries [3]:

$$
\sigma_{\varepsilon}=\sigma_{0 \varepsilon}+k_{\varepsilon} \ell^{-1 / 2}
$$

In Equation (1), $\sigma_{0 \varepsilon}$ is the ordinate axis intercept stress taken to apply for plastic flow within the grain volume, $k_{\varepsilon}$ is the microstructural stress intensity required for overcoming the grain boundary resistance, and $\ell$ is average grain diameter generally measured by a line intercept method. Very interestingly in Figure 1, the prominent metallurgist, Champion Mathewson, proposed that the hardness measurements could be approximated by an $\ell^{-1 / 4}$ dependence if the hardness was required to be zero for a single crystal. Otherwise, the finite ordinate intercept, $\sigma_{0 \varepsilon}$, has been correlated in a number of cases with single crystal plastic flow stress measurements.

\section{Nanopolycrystal Hall-Petch Grain Size Strengthening}

Many experimental and theoretical investigations have been reported on H-P dependence [4]. Current interest centers on the achievement of an order of magnitude increase in yield strength, that is achieved at nano-scale grain size dimensions. Figure 2 provides a $\log / \log$ representation of conventional and nano-polycrystalline H-P measurements following Equation (1) for copper, nickel, and aluminum, also at different values of strain [5]. An approximate order of magnitude grain size strengthening effect is observed. The indicated low $k_{0.14}$ value for nickel at large strain and conventional grain sizes, compared to the nano-scale $k_{\varepsilon}$ obtained from hardness measurements, is an anomalous result. At smaller proof strains, nickel $k_{\varepsilon}$ has been shown to be near to that of copper.

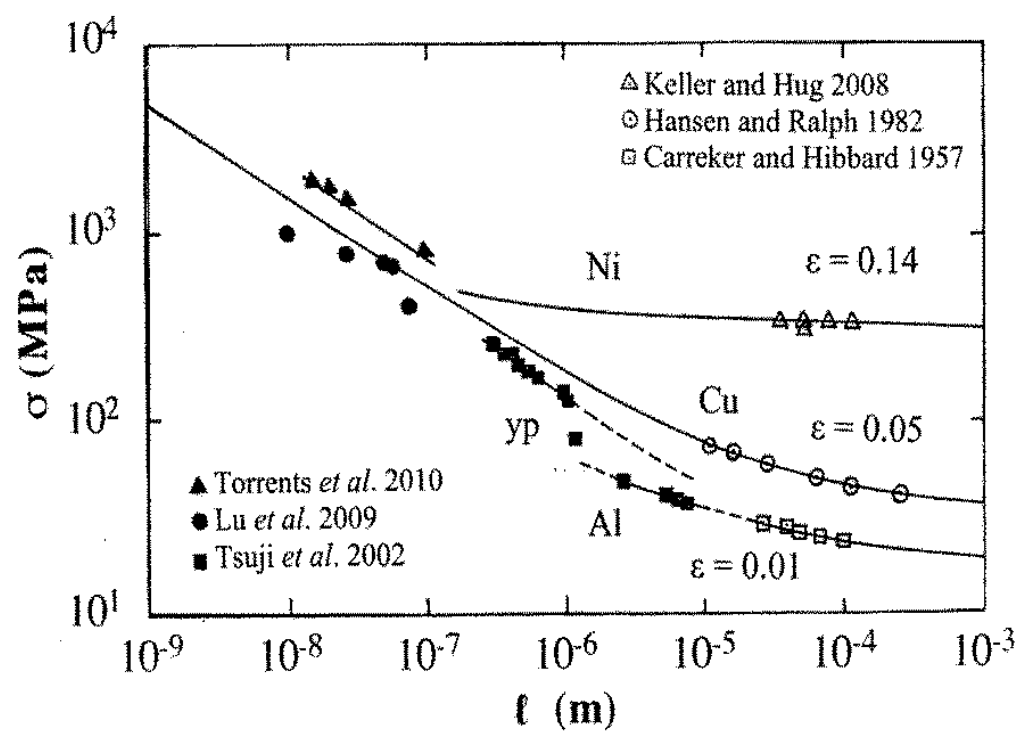

Figure 2. Comparison of Hall-Petch grain size dependent strengthening results at conventional and nano-polycrystalline grain sizes for $\mathrm{Al}, \mathrm{Cu}$, and $\mathrm{Ni}$ materials; the referenced data are given in Reference [5]. 
The near-equivalence of copper and nickel $k_{\varepsilon}$ values, along with a significantly lower value for aluminum, has been explained in terms of the calculated shear stress at the pile-up tip, $\tau_{C}$, being correlated with the need for cross-slip in effecting transmission of plastic flow across grain boundaries [5]; see Equation (2):

$$
k_{\varepsilon}=m_{\mathrm{T}}\left[\pi m_{\mathrm{S}} G b \tau_{\mathrm{C}} / 2 \alpha^{\prime}\right]^{1 / 2}
$$

In Equation (2), $m_{\mathrm{T}}$ and $m_{\mathrm{S}}$ are Taylor and Sachs orientation factors, $G$ is shear modulus, $b$ is Burgers vector, and $\alpha^{\prime} \approx 0.8$ is for an average dislocation character. A nearly equivalent numerical value of $G b \tau_{C}$ is obtained for copper and nickel, thus explaining their nearly same $k_{\varepsilon}$ values and, also, is consistent for aluminum with observation of a much lower $k_{\varepsilon}$ value controlled by easy cross-slip. The indicated increase in $k_{\varepsilon}$ in Figure 2 for aluminum when exhibiting a well-defined yield point, $(y p)$, is correlated also with the well-established measurement of a much larger $k_{\mathrm{yp}}$ for yield point behavior, for example, in steel.

\section{Thermally-Activated Dislocation Mobility Relations}

Jeffries reported in 1919 pioneering measurements on the combination of grain size, temperature, and strain rate dependencies of the mechanical properties of annealed and deformed copper materials [6]. A considerable number of other reports have followed on the topic, particularly involving the deformation of metal single crystals first produced during the same beginning period of the 20th century. Seeger reported in 1958 a summary description of fcc crystal deformation properties in terms of thermally-activated dislocation motion [7]. The report was followed in 1973 by the inclusion of an H-P dependence for polycrystals [8]. The single crystal/polycrystal topic was reviewed in 2008, with emphasis given to constitutive relations developed for deformation dynamics calculations under condition of high rate loading [9].

\subsection{Thermally-Activated FCC Strain Hardening}

The thermal dependence is in the strain hardening, $d \sigma_{\varepsilon} / d \varepsilon$, for fcc metals and alloys. One of several dislocation mechanics based constitutive equations proposed for $\sigma_{\varepsilon}$ is given by [10]:

$$
\sigma_{\varepsilon}=\sigma_{G \varepsilon}+B_{0}\left\{\varepsilon_{\mathrm{r}} \cdot\left[1-\exp \left(-\varepsilon / \varepsilon_{\mathrm{r}}\right)\right]\right\}^{1 / 2} \exp (-\alpha T)+k_{\varepsilon} \ell^{-1 / 2}
$$

In Equation (3), $\sigma_{G \varepsilon}$ is an athermal stress for elastic interactions within the polycrystal grain volumes, $\varepsilon_{\mathrm{r}}$ is a reference strain for dynamic recovery, and $\alpha=\alpha_{1}-\alpha_{2} \ln (\mathrm{d} \varepsilon / \mathrm{d} t)$ is a temperature coefficient including strain rate, $(\mathrm{d} \varepsilon / \mathrm{d} t)$, dependence that is rooted in the thermally-activated dislocation rate description. The first two terms are included within $\sigma_{0 \varepsilon}$ in Equation (1). At small $\varepsilon$ values, $\sigma_{\mathcal{\varepsilon}}$ follows a parabolic Taylor-type strain dependence.

An example calculation employing Equation (3) to describe the deformation shape of an impacted solid cylinder in comparison with the experimental shape is shown in Figure 3. The computed deformation shape, obtained with use of separately determined material constants from reference stress-strain tests, was achieved with the Elastic Plastic Impact Calculation (EPIC) code [11]. A slight improvement in the calculated deformation profile was obtained over another calculation applied to the same test result employing the eponymous Johnson-Cook numerical equation developed jointly with invention of the EPIC code. 


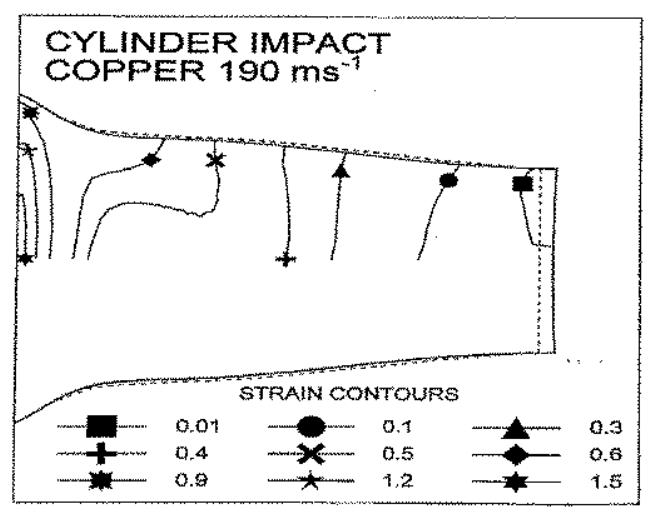

Figure 3. Comparison of calculated (continuous curve) and (dotted) experimental shapes for a longitudinal section of an impacted copper solid cylinder, including internal iso-strain profiles [10].

\subsection{Thermal BCC Yield Stress}

For bcc metals and alloys, the thermal dependence is in the lower yield point stress, $\sigma_{\text {lyp }}=\sigma_{\varepsilon}$, and the strain hardening is athermal. The counterpart constitutive equation for the behavior is given in Reference [10]:

$$
\sigma_{\varepsilon}=\sigma_{G \varepsilon}+B \exp (-\beta T)+A \varepsilon^{n}+k_{\varepsilon} \ell^{-1 / 2}
$$

In Equation (4), $\beta=\beta_{0}-\beta_{1} \ln (\mathrm{d} \varepsilon / \mathrm{d} t)$ and $A$ and $n$ are experimental constants describing a power law dependence for the strain hardening and the other parameters are the same as defined in Equation (3). Figure 4 shows an example deformation shape for an Armco iron solid cylinder impacted in the same manner as was done for copper in Figure 3, which the result has included the additional complication of deformation by twinning in the early stage of impact [11].

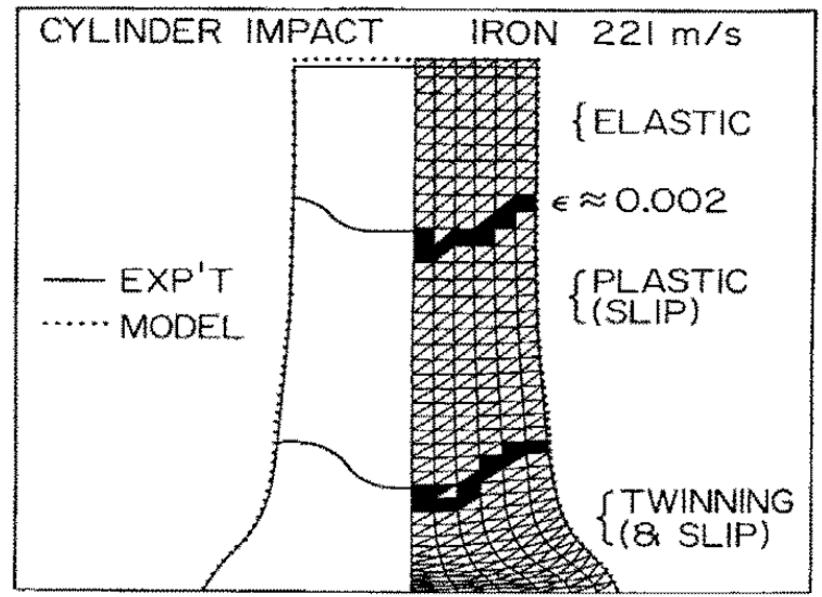

Figure 4. Experimental and Elastic Plastic Impact Calculation (EPIC) modeled solid cylinder impact test result on Armco iron as a result of initial athermal deformation twinning then followed by thermally-activated slip [10].

A previous report on solid cylinder impact tests made on $\alpha$-iron material had revealed in the region close to the impact surface the occurrence of deformation twins, then called Neumann bands, after their observation in meteorites [12]. Sequential EPIC calculations applied to the result shown in Figure 4 revealed that a limited amount of essentially athermal twinning occurred first on impact and hardened the material, in part, by grain size reduction, and then further deformation followed afterward by thermally-activated slip. Such twinning is known to follow an H-P dependence with constants, $\sigma_{0 \mathrm{~T}}<\sigma_{0 \varepsilon}$ and $k_{\mathrm{T}}>k_{\varepsilon}$, thus indicating a transition at smaller grain size when total deformation by slip 
is preferred to twinning. The profile of Figure 4 was shown to be essentially identical to the originally reported longitudinal section view containing the Neumann band structure [10,12].

\section{Brittle Fracturing and Fracture Mechanics}

At lower temperatures or higher plastic strain rates, brittle cleavage fracturing intervenes in tensile tests of steel and related bcc metals and alloys. The tensile cleavage fracture stress also follows an H-P dependence with a higher value of $k_{\mathrm{C}}>k_{\mathrm{T}}$. The characteristic temperature, $T_{\mathrm{C}}$, for the transition in behavior has been modeled on a dislocation mechanics basis [13]. The topic also relates importantly to the sudden onset of brittle failure that may occur due to the presence of a sharp crack, as included in the subject of fracture mechanics.

\subsection{The Ductile-to-Brittle Transition Temperature (DBTT)}

The brittleness transition behavior is depicted in Figure 5 for a compilation of measurements made on two steel materials with different grain sizes, and including measurements made of tensile yield stress, brittle fracture stresses in bend tests, and Charpy v-notch impact energy tests [13]. In the figure, the effective yield stress in the Charpy test has been raised by a notch factor, $\alpha=1.94$, to take account of the influence of hydrostatic component of stress and a small value of $\beta$ has been employed (appropriate to an effective strain rate of $400 \mathrm{~s}^{-1}$ ); see Equation (4). The effective H-P $\mathrm{k}_{\mathrm{lyp}}$ associated with the difference in yield stresses for the two grain sizes is seen to be a much smaller effect than the corresponding larger effect of $k_{\mathrm{C}}$ on the fracture stress, $\sigma_{\mathrm{C}}$, so producing a lower value of transition temperature for the smaller grain size material. The predicted transition for the smaller grain size was found to be raised somewhat because of easier cracking associated with the presence of carbide plates at the grain boundaries.

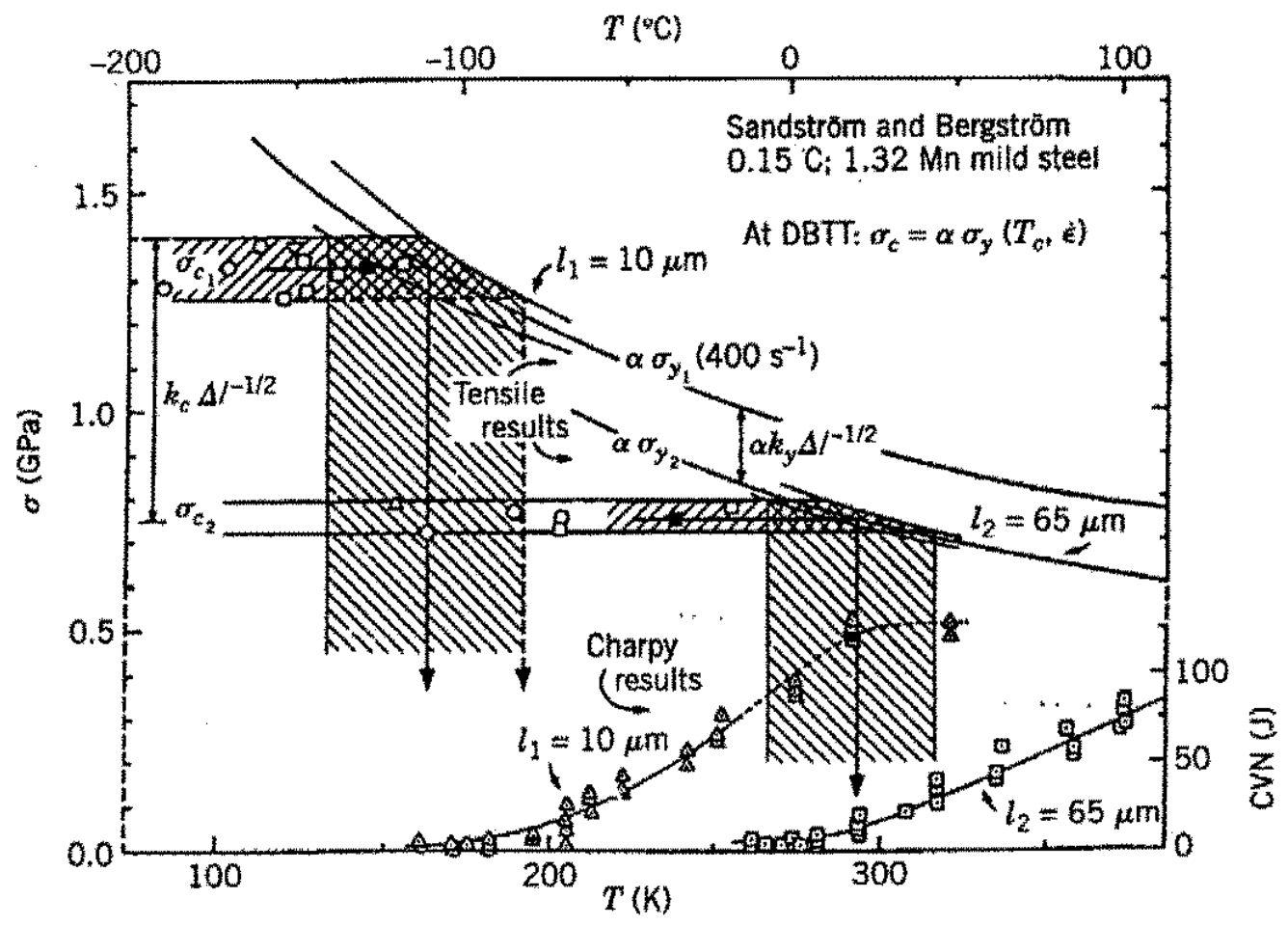

Figure 5. The ductile-to-brittle transition temperatures for two steel materials with different grain sizes of 65 and $10 \mu \mathrm{m}$ as determined in tensile tests and via Charpy v-notch (CVN) impact tests [13].

\subsection{Plastic Zone and Grain Size in Fracture Mechanics}

The notch effect in relatively small-scale Charpy impact tests relates to the role of crack size in fracture mechanics tests at micro- to macro-scale dimensions, and to the progression from Griffith's 
pioneering work on a reciprocal square root of crack size dependence for the fracture stress as extended on a continuum mechanics basis by Irwin [14]. The importance of the plastic zone size in the Charpy test is not obviated by any cracks, no matter how sharp, which are able to be put into a fracture mechanics test specimen [15]. Bilby, Cottrell, and Swinden employed a continuum dislocation pile-up model both for a crack and strip-type plastic zone at the crack tip [16]. The transcendental equation obtained for critical growth of the crack was shown to be closely approximated by the relationship [17]:

$$
\sigma_{\mathrm{F}}=A \sigma_{y}[s /(c+s)]^{1 / 2}
$$

In Equation (5), $A$ is a numerical constant near unity, $c$ is the half-length of an internal crack, and $s$ is the length of the plastic strip. Figure 6 shows application of the relationship with $A=1.0$ to measurements recently reported for the American Society for Testing and Materials (ASTM) specified fracture mechanics measurements made on AISI 1040 steel material [18].

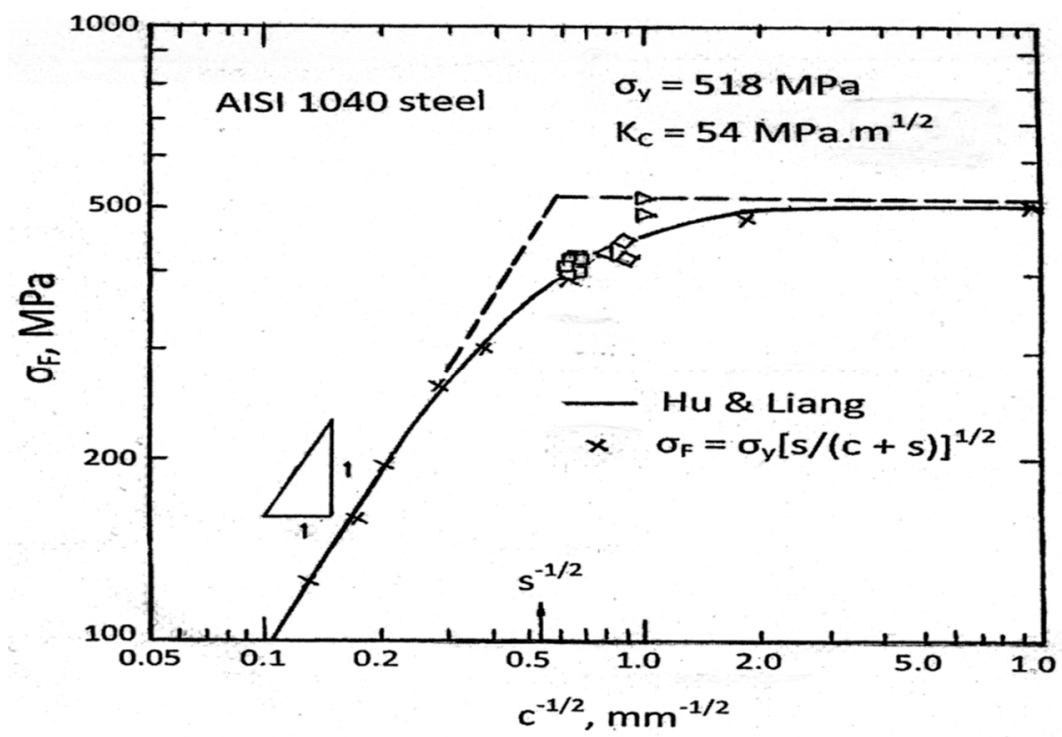

Figure 6. Comparison of fracture mechanics specified stress dependence on crack size for AISI 1040 steel obtained from results reported by $\mathrm{Hu}$ and Liang and matched with calculated plastic zone, $s$.

The extended continuous curve shown in Figure 6 was established by $\mathrm{Hu}$ and Liang for an AISI 1040 plate material of length $40 \mathrm{~mm}$ including crack size to length ratios between 0.1 and 0.7 , and with the separate measurements indicated for the yield stress and plane strain determined stress intensity, $K_{\mathrm{IC}}$, value. At large crack size, a linear dependence of fracture stress, $\sigma_{\mathrm{F}}$, on the reciprocal square root of crack size is obtained, as predicted. The indicated fit of $x$-marked points on the curve were obtained with Equation (5), with $s=3.5 \mathrm{~mm}$ that corresponds in the $\mathrm{Hu}$ and Liang calculation of a critical reference crack length of $4.04 \mathrm{~mm}$. A deviation from the predicted linear fracture mechanics relationship is seen to occur at relatively smaller $(c / s)<\sim 3$ than might have been expected, for example, proceeding onward from $\sigma_{\mathrm{F}} \geq 0.5 \sigma_{y}$.

The yield stress dependence in the fracture mechanics description has been extended in the same type analysis to description of the fracture mechanics stress intensity dependence on grain size in the relationship:

$$
K_{\mathrm{IC}}=(8 / 3 \pi)^{1 / 2}\left[\sigma_{0 \mathrm{C}}+k_{\mathrm{C}} \ell^{-1 / 2}\right] s^{1 / 2}
$$

In many cases, $s$ is relatively constant and therefore $K_{\mathrm{IC}}$ follows an H-P type dependence [15].

\section{Discussion}

The examples given of dislocation mechanics based relationships for hardness, fcc and bcc plastic flow stresses, impact, and fracturing properties constitute only a relatively limited number 
of connections being currently researched for a wide variety of metals and their alloys. An example is provided by hardness measurements being investigated in micro- and nano-indentation tests of nano-dimensional grain size nickel materials [5], and such measurements are being extended to indentation fracture mechanics measurements made on relatively more brittle materials [19].

In addition, the very positive influence of grain size on strengthening metals is being investigated in terms of a variety of material processing methods, in particular, by the method of severe plastic deformation (SPD) [20]. Both an increase in $\sigma_{0 \varepsilon}$ and decrease in $\ell$ contribute to increasing $\sigma_{\varepsilon}$. The method has historical connection with wire drawing of patented (eutectoid) steel wire known as piano wire, and which material is more recently being employed in the strengthening of automotive tires at nano-scale iron and iron carbide phase separations [21].

For fcc and hcp metals, there is an important magnification of the plastic strain rate sensitivity measured at nano-scale dimensions in that the pile-up stress, $\tau_{C}$, in Equation (2) is sufficiently small as to be affected by thermal activation, thus producing a grain size dependence for the activation area, $A^{*}=v^{*} / b=\left(k_{\mathrm{B}} T / b\right)\left(\Delta \ln [\mathrm{d} \varepsilon / \mathrm{d} t] / \Delta \tau_{\mathrm{Th}}\right)_{T}$ in which $v^{*}$ is the frequently employed activation volume, $k_{\mathrm{B}}$ is the Boltzmann constant, and $\tau_{\mathrm{Th}}=\sigma_{\mathrm{Th}} / m_{\mathrm{T}}$ is strain dependent. As a consequence, $\left(1 / v^{*}\right)$ follows a H-P type dependence:

$$
\left(1 / v^{*}\right)=\left(1 / v_{0}^{*}\right)+\left(k_{\varepsilon} / 2 m_{T} \tau_{C} v_{C}^{*}\right) \ell^{-1 / 2}
$$

In Equation (7), $\left(1 / v^{*}\right)$ applies to strain rate sensitivity within the polycrystal grain volumes, and $\tau_{\mathrm{C}} v^{*} \mathrm{C} \approx \tau_{\mathrm{CTh}} v^{*} \mathrm{C}$ is constant [5]. At very small grain sizes, say $<20 \mathrm{~nm}$, there is a reversal in the H-P dependence but the value of $v^{*}$ is substantially decreased even more to a size of atomic dimensions, coincident with grain size weakening attributed to atomic diffusion mechanisms.

Important strain rate sensitivity is involved also in the ductile-brittle transition behavior described in connection with Figure 5, as is true for the important influence of grain size dependence. However, greater emphasis is given normally to specifying as accurately as possible the fracture mechanics stress intensity parameter, $K_{\mathrm{IC}}$, employed to characterize the propensity of the material for the sudden onset of catastrophic failure. In this case, hardness testing again provides a useful method of characterizing the indentation fracture mechanics properties of relatively more brittle materials [19].

\section{Summary}

A brief description has been given of hardness, grain size, flow stress, temperature, strain rate, and crack size aspects of dislocation mechanics based descriptions of metal plasticity and fracturing. The purpose of the description has been to provide several examples among the many investigations already reported or being underway, in order to characterize the corresponding mechanical properties of metals and their alloys.

Conflicts of Interest: The author declares no conflict of interest.

\section{References}

1. Armstrong, R.W. Plasticity: Grain size effects III. In Reference Module in Materials Science and Engineering; Hashmi, S., Ed.; Elsevier: New York City, NY, USA, 2018; pp. 1-23.

2. Jindal, P.C.; Armstrong, R.W. The dependence of the hardness of cartridge brass on grain size. Trans. TMS-AIME 1967, 239, 1856-1857.

3. Armstrong, R.W. The influence of polycrystal grain size on several mechanical properties. Metall. Trans. 1970, 1, 1169-1176. [CrossRef]

4. Armstrong, R.W. 60 years of Hall-Petch: Past to present nano-scale connections. Mater. Trans. 2014, 55, 2-12. [CrossRef]

5. Armstrong, R.W. Hall-Petch description of nanopolycrystalline $\mathrm{Cu}, \mathrm{Ni}$ and $\mathrm{Al}$ strength levels and strain rate sensitivities. Phil. Mag. 2016, 96, 3097-3108. [CrossRef]

6. Jeffries, Z. Effect of temperature, deformation and grain size on the mechanical properties of metals. Trans. TMS-AIME 1919, 60, 474-576, with discussion by C.H. Mathewson and others. 
7. Seeger, A. Kristallplastizität. In Handbuch der Physik VII/2, Crystal Physics II; Flugge, S., Ed.; Springer: Berlin, Germany, 1958; pp. 1-210.

8. Armstrong, R.W. Thermal activation-strain rate analysis (TASRA) for polycrystalline materials. J. Sci. Ind. Res. 1973, 32, 591-598.

9. Armstrong, R.W.; Walley, S.M. High strain rate properties of metals and alloys. Intern. Mater. Rev. 2008, 53, 105-128. [CrossRef]

10. Zerilli, F.J.; Armstrong, R.W. Dislocation mechanics based analysis of material dynamics behavior: Enhanced ductility, deformation twinning, shock deformation, shear instability, dynamic recovery. J. Phys. IV France Colloq. 1997, 7, 637-642. [CrossRef]

11. Johnson, G.R.; Cook, W.H. A constitutive model and data for metals subjected to large strains, high strain rates, and high temperatures. In Proceedings of the 7th International Symposium on Ballistics, The Hague, The Netherlands, 19-21 April 1983; pp. 541-547.

12. Carrington, W.E.; Gaylor, M.L.V. The use of flat-ended projectiles for determining dynamic yield stress III. Changes in microstructure caused by deformation under impact at high striking velocities. Proc. R. Soc. Lond. A 1948, 194, 323-331.

13. Armstrong, R.W. Material grain size and crack size influences on cleavage fracturing. Phil. Trans. R. Soc. A 2015, 373, 20140124. [CrossRef] [PubMed]

14. Irwin, G.R. Fracture. In Handbuch der Physik VI; Flugge, S., Ed.; Springer: Berlin, Germany, 1958; pp. 551-590.

15. Armstrong, R.W. Crack Size and Grain Size Dependence of the Brittle Fracture Stress. In Dritte Intern. Tagung uber den Bruck, ICF3; Kochendorfer, A., Ed.; Verein Deutscher Eisenhuttenleute: Dusseldorf, Germany, 1973; p. III-421.

16. Bilby, B.A.; Cottrell, A.H.; Swinden, K.H. The Spread of Plastic Yield from a Notch. Proc. R. Soc. Lond. A 1963, 272, 304-314.

17. Armstrong, R.W. Dislocation viscoplasticity aspects of material fracturing. Eng. Fract. Mech. 2010, 77, 1348-1359. [CrossRef]

18. Hu, X.-Z.; Liang, L. Elastic-Plastic and Quasi-Brittle Fracture. In Handbook of Mechanics of Materials; Hsueh, C.H., Schmauder, S., Chen, C.-S., Chawla, K.K., Chawla, N., Chen, W., Kagawa, Y., Eds.; Springer: Singapore, 2019; pp. 1-32, see Figure 11.

19. Armstrong, R.W.; Walley, S.M.; Elban, W.L. Elastic, plastic and cracking aspects of the hardness of materials. Int. J. Mod. Phys. B 2013, 28, 1330004. [CrossRef]

20. Vinogradov, A.; Estrin, Y. Analytical and numerical approaches to modelling severe plastic deformation. Prog. Mater. Sci. 2018, 95, 172-242.

21. Armstrong, R.W. Size Effects on Material Yield Strength/Deformation/Fracturing Properties. J. Mater. Res. 2019, in press. [CrossRef] 